\title{
A Comparative Test of Brillouin Amplification and Erbium-doped Fiber Amplification for the Generation of Millimeter Waves with Low Phase Noise Properties
}

\author{
Markus Junker \\ Technological University Dublin \\ Max Ammann \\ Technological University Dublin, max.ammann@tudublin.ie \\ Andreas Schwarzbacher \\ Technological University Dublin, andreas.schwarzbacher@tudublin.ie
}

See next page for additional authors

Follow this and additional works at: https://arrow.tudublin.ie/engscheceart

Part of the Computer Engineering Commons

\footnotetext{
Recommended Citation

TMTT.2006.871321

This Article is brought to you for free and open access by the School of Electrical and Electronic Engineering at ARROW@TU Dublin. It has been accepted for inclusion in Articles by an authorized administrator of ARROW@TU Dublin. For more information, please contact arrow.admin@tudublin.ie, aisling.coyne@tudublin.ie, gerard.connolly@tudublin.ie.
}

Junker, M. et al. (2006) A comparative test of Brillouin amplification and Erbium-doped fiber amplification for the generation of millimeter waves with low phase noise properties. IEEE Transactions on Microwave Theory and Techniques, June 2006,Volume: 54 , no. 4 , Part 1, 1576 - 1581. doi:10.1109/

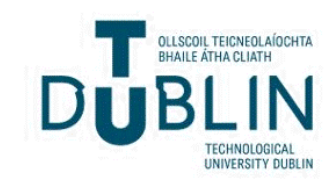


Authors

Markus Junker, Max Ammann, Andreas Schwarzbacher, Jens Klinger, Kai-Utwe Lauterback, and Thomas Schneider 


\section{A Comparative Test of Brillouin Amplification and Erbium-Doped Fiber Amplification for the Generation of Millimeter Waves With Low Phase Noise Properties}

Markus Junker, Max James Ammann, Member, IEEE, Andreas Thomas Schwarzbacher, Jens Klinger, Kai-Uwe Lauterbach, and Thomas Schneider

\begin{abstract}
Measurements of phase noise of a 40-GHz carrier signal are presented. The carrier is generated by the amplification of harmonics due to stimulated Brillouin scattering. An analogy to a generation of millimeter waves by an erbium-doped fiber instead of the Brillouin amplifier is investigated and discussed. In our setup, both show a comparable behavior in respect to their noise characteristics.
\end{abstract}

Index Terms-Brillouin scattering, millimeter-wave generation, phase noise.

\section{INTRODUCTION}

M ILLIMETER waves are expected to be promising and important frequencies for future wireless communication systems. The frequency domain above $30 \mathrm{GHz}$ offers an increase of the transmission bandwidth for radio links. The millimeter-wave frequency band overcomes the spectral congestion in lower frequency regions and offers an enormously large bandwidth. In the 60-GHz band, for instance, data transmission of $1.25 \mathrm{Gbit} / \mathrm{s}$ was shown [1]. Furthermore in [2], a wireless link with a data rate up to $3 \mathrm{Gbit} / \mathrm{s}$ and a carrier frequency of $120 \mathrm{GHz}$ was verified. Short-range propagation systems in the 59-64-GHz frequency band that is set as a target frequency band has been developed [3]-[5] in Japan.

The requirements for carrier frequencies in wireless communications are, in fact, very high. Beside frequency stability, magnitude, and a narrow bandwidth, the most important property of a carrier signal is the phase noise. It describes the noise of the phase at a certain offset from the carrier measured in

Manuscript received October 10, 2005; revised December 8, 2005.

M. Junker is with the School of Electronic and Communications Engineering, Dublin Institute of Technology, Dublin 8, Ireland and also with the Deutsche Telekom Fachhochschule Leipzig, 04275 Leipzig, Germany (e-mail: junker@fh-telekom-leipzig.de).

M. J. Ammann is with the Centre for Telecommunications ValueChain-Driven Research, School of Electronic and Communications Engineering, Dublin Institute of Technology, Dublin 8, Ireland (e-mail: max.ammann@dit.ie).

A. T. Schwarzbacher is with the School of Electronic and Communications Engineering, Dublin Institute of Technology, Dublin 8, Ireland (e-mail: andreas. schwarzbacher@dit.ie).

J. Klinger, K.-U. Lauterbach, and T. Schneider are with the Deutsche Telekom Fachhochschule Leipzig, 04275 Leipzig, Germany (e-mail: klinger@ fh-telekom-leipzig.de; kul@ ostec.org; schneider@fh-telekom-leipzig.de).

Digital Object Identifier 10.1109/TMTT.2006.871321 a $1-\mathrm{kHz}$ bandwidth $(\mathrm{dBc} / \mathrm{Hz})$. It limits the application of carrier signals and is an important criterion for the use of different modulation formats in communication systems [6]. Phase noise degrades millimeter and microwave systems introducing a significant irreducible error rate. In [7], the $60-\mathrm{GHz}$ region phase noise of -59 and $-85 \mathrm{dBc} / \mathrm{Hz}$ at 10 and $100 \mathrm{kHz}$, respectively, offset from the carrier were measured. A millimeter-wave generation of $42 \mathrm{GHz}$ was shown in [8] where a measurement of $-80 \mathrm{dBc} / \mathrm{Hz}$ at $10-\mathrm{kHz}$ offset was verified. By using the method of PM-IM conversion in chirped fiber gratings, a phase noise of $-87 \mathrm{dBc} / \mathrm{Hz}$ at $10-\mathrm{kHz}$ offset at a frequency of $28 \mathrm{GHz}$ were measured [9]. Currently, Kawanishi et al. [10] reported on a 44-GHz signal generated by an integrated reciprocating optical modulator with a phase noise property of $-88.5 \mathrm{dBc} / \mathrm{Hz}$ at $10-\mathrm{kHz}$ offset from the carrier. In [11], the millimeter-wave generation of $33.87 \mathrm{GHz}$ was realized by using hybrid mode locking of a monolithic distributed Bragg reflector (DBR) laser and a phase noise of $-70 \mathrm{dBc} / \mathrm{Hz}$ was measured.

In this paper, we focus our discussions on the low phase-noise properties of the Brillouin amplification part. This gain can be seen as one of the most significant parts of signal generation in our system. This noise property is proven by a comparison to an erbium-doped fiber (EDF) amplification. It is realized by replacing the Brillouin amplifier in the setup physically. An alignment of all parameters to both amplification versions makes this possible.

In Section II, the experimental setup is briefly described. Theoretical results of the low phase-noise analyses are shown in Section III. In Section IV, experimental results focused on a comparison of Brillouin amplification and EDF amplification are evaluated. Finally, the conclusions and discussions are given in Section V.

\section{EXPERIMENT}

Stimulated Brillouin scattering (SBS) is the nonlinear effect with the smallest threshold. SBS is an interaction between the material (optical fiber) and the incident light wave. The result is a density modulation of the refractive index, which can be seen as an acoustic wave. The pump wave is scattered at the grating resulting in a backward scattered wave, i.e., the Stokes wave. Owing to the Doppler effect, the Stokes wave is down shifted in frequency of $10.7 \mathrm{GHz}$. We measured a Brillouin gain bandwidth of $28.8 \mathrm{MHz}$. A similar experimental setup was discussed 


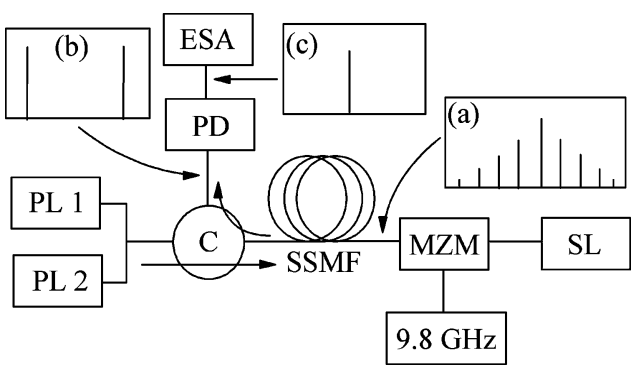

Fig. 1. Experimental setup for the generation of millimeter waves. (SL: signal laser, MZM: Mach-Zehnder modulator, PL: pump laser, PD: photodiode, ESA: electrical spectrum analyzer, C: circulator, SSMF: standard single-mode fiber.)

in detail in [12]. At this point, we want to give an overview about the system idea. A fiber laser is used as the signal laser with a narrow linewidth $<2 \mathrm{kHz}$ (Fig. 1), 20-mW output power, and a wavelength of $1550 \mathrm{~nm}$. The linewidth of the signal laser should be as low as possible owing to the fact that it significantly defines the linewidth of the millimeter-wave signal. A polarization controller adjusts the polarization to its optimum for the Mach-Zehnder modulator (MZM). External modulation by an MZM driven in the nonlinear domain (upper/lower quadratic operation point) is performed to generate a frequency comb. The modulation frequency is $9.8 \mathrm{GHz}$, hence, the separation of the sidebands has a distance of $9.8 \mathrm{GHz}$ [see Fig. 1, inset (a)]. By changing the operation point, switching between even- and odd-order sidebands is possible. The frequency comb is injected into a 50.43-km standard single-mode fiber (SSMF). The light of two DFB pump lasers combined in a 3-dB coupler is launched into the same fiber from the opposite side via a circulator. The frequencies of the pump lasers are adjusted in such a manner that the frequencies are $10.7 \mathrm{GHz}$ higher than the frequencies in the comb. Due to this, only two wavelengths out of the comb are amplified by Brillouin scattering. The pump power is below the Brillouin threshold that we calculated to be around $8.1 \mathrm{~mW}$. Hence, the Stokes wave is stimulated by the sideband and not by the noise in the fiber. The linewidth of the pump lasers is below $1 \mathrm{MHz}$.

Due to the natural attenuation, all other sidebands are attenuated while propagating in the fiber. The result at the output of the circulator is two strong frequency components with a separation depending on the order of the amplified sidebands: 19.6, 39.2, $58.8 \mathrm{GHz}$ and so on [see Fig. 1, inset (b)]. The two amplified harmonics are then superimposed in a photodiode (PD) and the actual millimeter wave is generated by heterodyning [see Fig. 1, inset (c)]. The optical input power at the PD is $1 \mathrm{~mW}$. The output frequency is $2 n f$ with $f$ being the frequency of the electrical generator and $n$ being the order of the sideband.

The modulation of the millimeter wave can be done quite simply by modulating the electrical generator, the signal laser, one of the pump lasers, or one amplified sideband.

\section{THEORY}

One of the simplest ways of generating millimeter waves is the heterodyning of two frequencies in a PD. The generation of two phase correlated waves can be done by double-sideband suppressed carrier (DSB-SC) modulation with MZMs. Due to the fact that both optical components are derived from a single source, their phases are totally correlated at the source [13]. If the optical component (sidebands) separation is in the tens of gigahertz region and fiber length of many tens of kilometers, the decorrelation effect owing to the fiber dispersion is negligible small. Hence, the millimeter-wave signal has a minimum of phase noise induced by the two heterodyned waves. In principle, the millimeter-wave generation of our setup follows the same restrictions as the generation by the heterodyning of two sidebands of an SSB-SC modulation in a PD [13]. If one assume no Brillouin amplification process in our setup, the resulting spectrum and the phase noise is the same. The only alternation in our setup is the adding of a Brillouin amplification process. The theoretical conditions for limiting the phase-noise induction to the millimeter wave are described in the following.

SBS as an amplifier, in general, induces many problems to the system. The Brillouin gain bandwidth is low and the spontaneous emission noise (SEN) can be 500 times larger than in a Raman amplifier [14]. Furthermore, a noise figure (NF) of $20 \mathrm{~dB}$ limits the system performance for preamplifiers.

In contrast to that, Ferreira et al. [15] reported that driving the amplifier in the saturation regime could significantly reduce the SEN. A short amplifier length can provide considerable improvement on the gain of the signals and decreases the SEN as well. Furthermore, the noise power increases with signal detuning. Hence, if the detuning between the pump and signal wave is minimized, the noise power can be notably reduced. Another way for decreasing the amplified spontaneous noise power is the setting of relatively high input signals powers when the Brillouin amplifier is operated in the saturation regime.

To maintain the phase-correlated state during their propagation in the fiber and while amplifying by two independent lasers, one has to consider two properties of the system. They are described below. A basis for simulating the amplification of two frequencies was shown in [16] where nonlinear effects accompanied with the susceptibility $\chi^{(3)}$ and the phase shift due to Brillouin amplification were considered. A part of a differential equation system, which describes the amplified sidebands at the circulator output, is shown in (1) and (2) as follows:

$$
\begin{aligned}
\frac{\partial E_{S 2}}{\partial z}= & \frac{g_{B}}{2 A_{\mathrm{eff}}} \Delta k_{1} E_{s 2} P_{P 2}+\frac{\alpha}{2} E_{S 2} \\
& +j\left[\frac{g_{B}}{2 A_{\mathrm{eff}}} \Delta k_{2} E_{s 2} P_{P 2}\right. \\
& \left.\quad+\gamma\left(P_{S 2}+2 P_{P 1}+2 P_{P 2}+2 P_{S 1}\right) E_{s 2}\right] \\
\frac{\partial E_{S 1}}{\partial z}=- & \frac{g_{B}}{2 A_{\mathrm{eff}}} \Delta k_{1} E_{s 1} P_{P 1}+\frac{\alpha}{2} E_{S 1} \\
& +j\left[\frac{g_{B}}{2 A_{\mathrm{eff}}} \Delta k_{2} E_{s 1} P_{P 1}\right. \\
& \left.\quad+\gamma\left(P_{S 1}+2 P_{P 1}+2 P_{P 2}+2 P_{S 2}\right) E_{s 1}\right]
\end{aligned}
$$

where $P_{s}$ is the power of the signal wave, $P_{P}$ is the power of the pump wave, $\Delta k_{2}$ is the phase matching factor, $\alpha$ is the attenuation in the fiber, $\gamma$ is the nonlinear coefficient, and $A_{\mathrm{eff}}$ is the effective core area. Equations (1) and (2) show the first and 
second amplified sideband, respectively, with their amplification by SBS and the attenuation in the real part. The imaginary part (in squared brackets) describes the phase shift due to nonlinear processes accompanied by $\gamma$ like cross phase modulation (XPM) and self phase modulation (SPM) (in brackets). Furthermore, the phase shift due to the Brillouin gain coefficient $g_{B}$ is considered.

If we assume that the signal waves $\left(P_{S 1}=P_{S 2}\right)$ and pump waves $\left(P_{P 1}=P_{P 2}\right)$ have the same power, the phase shift due to SPM and XPM of both amplified sidebands is equal. Furthermore, the Brillouin gain $g_{B}$ and the effective area $A_{\text {eff }}$ have the same values in (1) and (2), respectively. Hence, the phase-matching factor $\Delta k_{2}$ is variable and is described by

$$
\Delta k_{2}=\frac{\Delta k}{\left(\frac{\alpha_{a}}{2}\right)^{2}+\Delta k^{2}}
$$

and

$$
\Delta k=\frac{1}{v_{a}\left(\omega_{s}-\omega_{s \mathrm{MAX}}\right)}
$$

where $v_{a}$ is the velocity and $\alpha_{a}$ is the attenuation coefficient of the acoustic wave. $\omega_{S}$ is the signal wavelength and $\omega_{s \mathrm{MAX}}$ is the wavelength for maximum amplification. If there is no detuning between the signal wave and pump wave $\left(\omega_{S}=\omega_{s \mathrm{MAX}}\right)$, the phase shifts of both amplified sidebands are equal [16].

Concluding the theoretical part: the influence of phase decorrelation of two waves derived from one source is negligible. A high signal power, a low detuning, a short fiber length, and driving the amplifier in the saturation regime can significantly reduce the noise. If the power of both sidebands is nearly equal, the phase change due to SPM and XPM is the same. If the detuning between the pump and the signal wave is minimal, the phase shifts for both sidebands are also equal. Hence, the SBS influences on the millimeter wave is small. All theoretical derived system and setup settings have been considered with the exception of the short fiber length.

\section{EXPERIMENTAL RESULTS}

In order to prove the high signal performance of the generated carrier, it is necessary to analyze the stability, spectrum, and phase-noise properties. Due to the fact that our equipment (spectrum analyzer and PD) is frequency limited to $40 \mathrm{GHz}$, all measurements have been done in this domain. Theoretically, the system (Fig. 1) is able to generate up to $160 \mathrm{GHz}$ depending on the MZM used in the setup. Other possible frequency comb-generation techniques would have only the bandwidth of the PD as a limitation, which is $330 \mathrm{GHz}$ [17]. This could be a Fabry-Perot laser or a comb generation due to four wave mixing (FWM). Fig. 2 shows the carrier signal at a frequency of $39.199989 \mathrm{GHz}$ with a bandwidth of $300 \mathrm{~Hz}$. Due to the resolution limitation of the electrical spectrum analyzer (ESA), it was not possible to measure the real bandwidth of the carrier. The ESA has a resolution bandwidth (RBW) and a video bandwidth (VBW) of $300 \mathrm{~Hz}$. Note that the actual bandwidth is possibly lower, but not

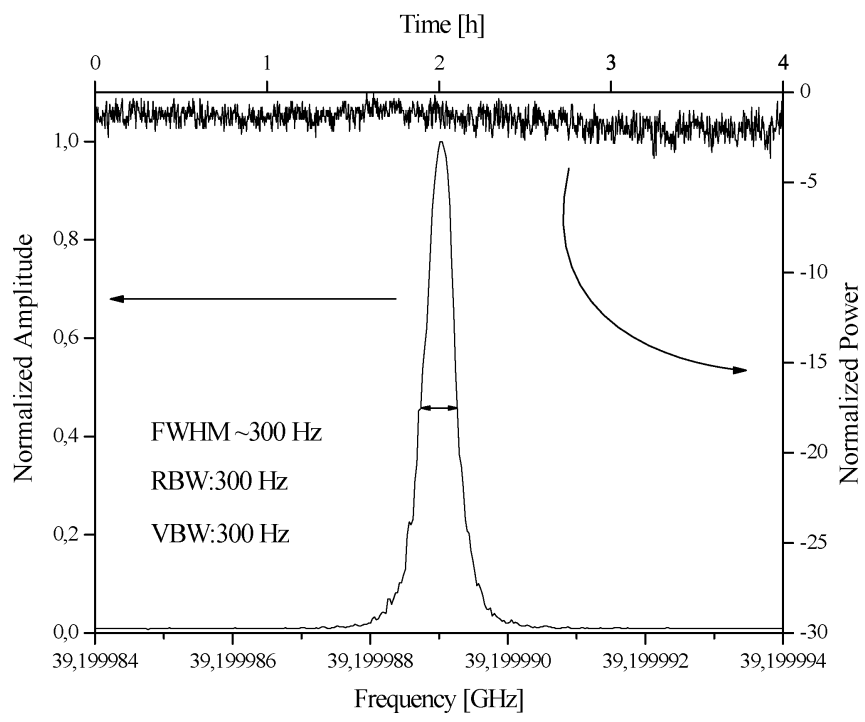

Fig. 2. Carrier signal at $39.199989 \mathrm{GHz}$ at the output of the PD (bottom and left) and long-term stability of itself (top and right).

measurable. The signal was measured with an ESA at the output of the PD. As one can see, it has very good spectral purity. The detuning of the signal wave and pump wave is minimized and the amplified sidebands have the same magnitude.

Fig. 2 shows also a power time measurement over a range of $4 \mathrm{~h}$ and it is proven that the signal is indeed constant. The short-term fluctuations in the plot are a result of the temperature controllers of the pump lasers. Due to a thermistor setting resolution of minimal $1 \Omega$, which is approximately $42 \mathrm{MHz}$, the frequency of the pump lasers changes within this range. This fluctuation causes a frequency shift of the Brillouin gain and, thus, a change of the amplitude of the amplified harmonic. Owing to the fact that two sidebands are amplified, the fluctuations are doubled. Distortions of this kind can be easily and significantly reduced by using temperature controllers with a higher setting resolution.

The temperature stability depends significantly on the components used in the system. The $9.8-\mathrm{GHz}$ generator has frequency stability of $< \pm 5 \cdot 10^{-8} \mathrm{~Hz} /{ }^{\circ} \mathrm{C}$. The pump laser wavelength is adjusted by a control loop to stay fixed at the wavelength of the sideband that should be amplified. The electro/optical frequency conversion in the MZM is temperature independent as well. The only parameter, which is a function of the temperature, is the Brillouin shift. In [18], it is shown that the Brillouin shift is $1.36 \mathrm{MHz} /{ }^{\circ} \mathrm{C}$ in an SSMF at $1.32 \mu \mathrm{m}$. If we assume that the Brillouin shift at $1.55 \mu \mathrm{m}$ is nearly equal and the Brillouin bandwidth is $28.8 \mathrm{MHz}$, there is a need of $\pm 10.6^{\circ} \mathrm{C}$ temperature change for a total detuning of the Stokes wave and sideband. Due to the fact that the detuning of both amplified sidebands would be the same, a temperature shift affects only the power of the millimeter wave, but not the phase. This problem can be reduced, on the one hand, by a control loop, which regulate the pump laser wavelength to the maximum amplification and, on the other hand, the Brillouin bandwidth can easily be broadened by an external phase modulation [19]. This increases the Brillouin bandwidth and, hence, the system independence on the temperature. Note that there was no connection 


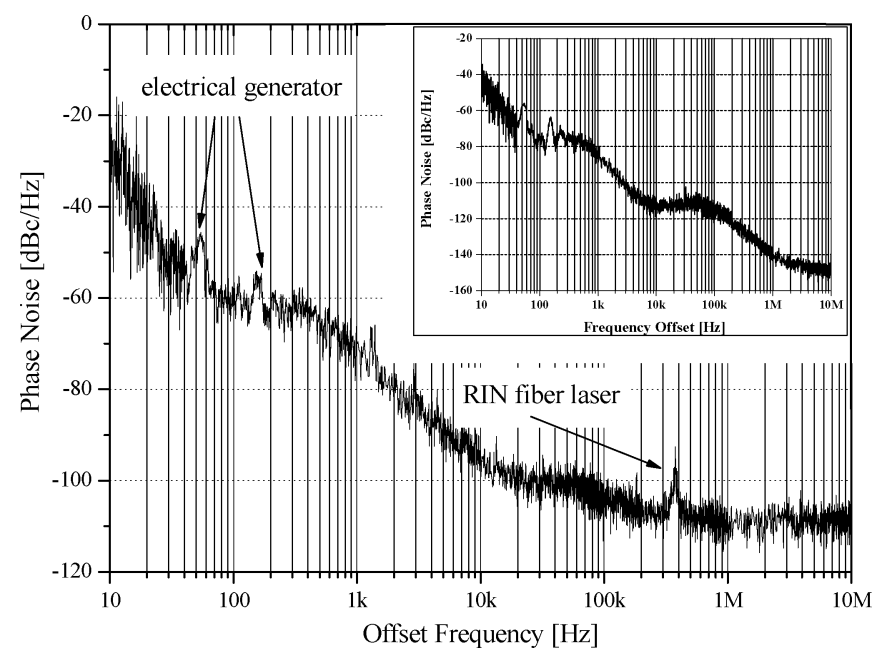

Fig. 3. Phase-noise measurement of the $39.199989-\mathrm{GHz}$ signal. At $10-\mathrm{kHz}$ offset from the carrier, the phase noise is $-94.8 \mathrm{dBc} / \mathrm{Hz}$. The inset shows the phase noise property of the $9.8-\mathrm{GHz}$ oscillator.

between the temperature and millimeter-wave frequency realized during the measurements.

The phase noise of the generated signal is shown in Fig. 3. The noise at a frequency separation above $10 \mathrm{kHz}$ from the carrier is $-94.8 \mathrm{dBc} / \mathrm{Hz}$, although the method is not yet optimized. This is a remarkably small value compared to other investigations on millimeter waves [7]-[11]. If one considers a Brillouin amplification process in the system, this result is even more impressive. Due to the inefficient opto-electronic conversion, the magnitude of the measured signal is $-26.5 \mathrm{dBm}$. The input power at the $\mathrm{PD}$ is $1 \mathrm{~mW}$. By optimizations of the fiber length shown in [15], one can assume a further decrease of the phase noise. The peaks at 55 and $150 \mathrm{~Hz}$ have their origin in the $9.8-\mathrm{GHz}$ signal generator, which provides the MZM with the necessary power. The total phase noise of the millimeter wave is induced, on the one hand, by the noise of each component used in the system and, on the other hand, the phase noise induced by the SBS has to be added logarithmically together with the noise of the components. The peak at $300 \mathrm{kHz}$ is the relative intensity noise (RIN) oscillation peak that is typically around $250-300 \mathrm{kHz}$ in the fiber laser (signal laser).

The generation of millimeter waves by Brillouin scattering (Fig. 1) is a closed system. Hence, the output signal is the object that needs to be analyzed. On the other hand, we want to show that SBS is still of interest as an amplifier for special applications. Therefore, it is necessary to evaluate the characteristics of the Brillouin amplifier.

An important property of conventional amplification systems is the NF. The NF is the ratio of actual output noise to that which would exist if the device itself did not introduce noise.

Due to the fact that all other devices in our system have an unknown phase noise portion, it is not possible to draw a conclusion from it to the Brillouin amplification part. The NF analysis of the Brillouin amplifier in our setup is only possible by a comparison test. Two setup changes are necessary to realize this comparison.

First we change the MZM operation point in such a manner that only even-order sidebands are generated (carrier, 2, 4, 6,

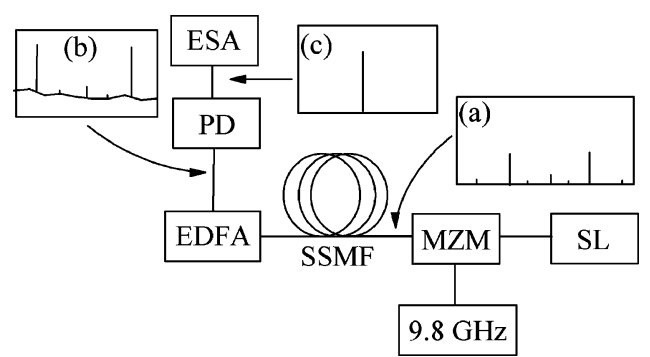

Fig. 4. Modified setup for comparison SBS-EDFA gain. (EDFA: erbium-doped fiber amplifier.)

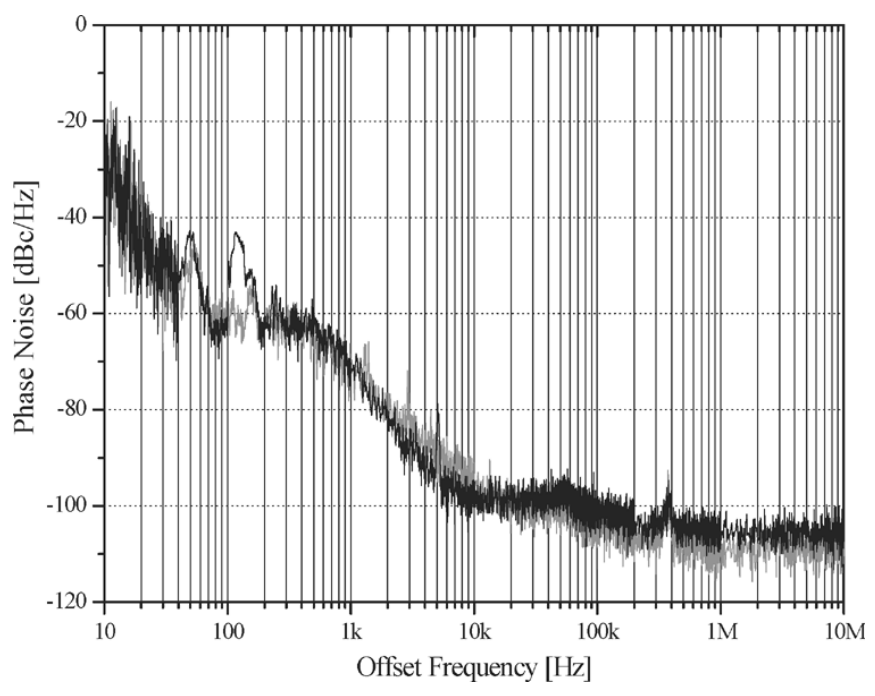

Fig. 5. Phase-noise measurement of the 39.199989-GHz signal generated by an EDFA (dark curve). At $10-\mathrm{kHz}$ offset from the carrier the phase noise is $-95 \mathrm{dBc} / \mathrm{Hz}$. The bright curve is phase-noise measurement of the 39.199989-GHz signal generated by SBS (see Fig. 3).

8) by changing the bias voltage [see Fig. 4, inset (a)]. Hence, all other harmonics are suppressed. The second harmonics have the highest magnitude and are adjusted in such a manner that they have the same value as before the change. Due to the natural attenuation in the 50-km fiber, the optical power is decreased. In order to have the same output power, there is a need to amplify the modified frequency comb with a gain of $32 \mathrm{~dB}$ by an erbiumdoped fiber amplifier (EDFA) [see Fig. 4, inset (b)]. The EDFA has a maximum NF of $6 \mathrm{~dB}$ at a gain of $32 \mathrm{~dB} .^{1}$ The optical power of the EDF amplified sidebands is $1 \mathrm{~mW}$, which is the same as in the measurement in Fig. 3. Hence, the optical input power at the $\mathrm{PD}$ is $1 \mathrm{~mW}$ as well. At the output of the $\mathrm{PD}$, again the 39.199989-GHz carrier signal is detected [see Fig. 4, inset (c)].

The amplification of the sidebands by an EDFA allows a comparison between the two amplification systems because all other parameters are unchanged. Although the phase spectrum at $10-\mathrm{kHz}$ offset is nearly equal $(-95 \mathrm{dBc} / \mathrm{Hz})$ to the SBS gained signal, the quality has decreased near the carrier.

As can be seen in Fig. 5, the influence of the electrical generator increases as one can see at $130 \mathrm{~Hz}$ due to the EDFA amplification. It proves that the Brillouin amplifier driven in our regime

\footnotetext{
${ }^{1}$ Personal contact with the EDFA manufacture/results of simulations.
} 
induces a low amplified spontaneous emission (ASE) noise to the signal, as does an EDFA.

We have shown that the impact to the phase of a signal by a Brillouin amplifier can be significantly reduced. We focus our future research to the modulation of the signal and the optimization in respect to the noise.

\section{CONCLUSION}

New phase-noise measurements of a millimeter waves have been presented. The values show very low phase-noise properties in the frequency domain at $40 \mathrm{GHz}$. A phase-noise value of $-94.8 \mathrm{dBc} / \mathrm{Hz}$ prove the high quality of the signal. This low phase noise is even more impressive if one considers Brillouin amplification in the setup. We also discussed the comparison between the application of a Brillouin amplifier and an EDF amplifier in our setup. It is realized by replacing the Brillouin amplifier with an EDF amplifier and aligning all parameters to the reference (EDFA). It shows a high-quality NF performance.

Although the EDF amplification has shown similar phase-noise results, it is unusable for the generation of millimeter waves up to $160 \mathrm{GHz}$. The EDF amplifies all sidebands in the same way, which limits the millimeter-wave frequency to $40 \mathrm{GHz}$ depending on the operation point of the MZM [see Fig. 4, inset (a)]. In our setup, we used the SBS as a filter as well. The selective Brillouin amplification amplifies just the sidebands that correspond to the desired carrier signal. An amplification of the eighth sideband is possible by SBS that is not using an EDFA. The EDF amplification is only a comparison parameter in this paper.

\section{ACKNOWLEDGMENT}

The authors would like to acknowledge the assistance of C. Schäffer and G. Staats, both of the Technische Universität Dresden, Dresden, Germany, and the help with the phase-noise measurements. The authors further acknowledge the donation of the phase-noise equipment by the Technische Universität Dresden.

\section{REFERENCES}

[1] K. Ohata, K. Maruhashi, M. Ito, S. Kishimoto, K. Ikuina, T. Hashiguchi, N. Takahashi, and S. Iwanaga, "Wireless $1.25 \mathrm{~Gb} / \mathrm{s}$ transceiver module at $60 \mathrm{GHz}$ band," in Proc. Int. Solid-State Circuits Conf., 2002, pp. 298-299.

[2] A. Hirata, M. Harada, and T. Nagatsuma, "120-GHz wireless link using photonic techniques for generation, modulation, and emission of millimeter-wave signals," J. Lightw. Technol., vol. 21, no. 10, pp. 2145-2153, Oct. 2003.

[3] T. Ihara and K. Fujimura, "Research and development trends of millimeter-wave short-range application systems," IEICE Trans. Commun., vol. E79-B, no. 12, pp. 1741-1753, Dec. 1996.

[4] T. Ihara, T. Manabe, M. Fujita, T. Matsui, and Y. Sugimoto, "Research activities on millimeter-wave indoor wireless communication system at CRL," in Proc. ICUPC'95, Tokyo, Japan, Nov. 1995, pp. 197-200.

[5] Y. Takimoto, "Recent activities on millimeter wave indoor LAN system development in Japan," in Proc. IEEE MTT-S Int. Microw. Symp. Dig., Orlando, FL, May 1995, vol. WE1D-3, pp. 405-408.

[6] M. Iqbal, J. Lee, and K. Kim, "Performance comparison of digital modulation schemes with respect to phase noise spectral shape," in Proc. Can. Electr. Comput. Eng. Conf., Mar. 2000, vol. 2, pp. 856-860.
[7] K. Kitayama, "Ultimate performance of optical DSB signal-based millimeter-wave fiber-radio system: Effect of laser phase noise," J. Lightw. Technol., vol. 17, no. 10, pp. 1774-1781, Oct. 1999.

[8] D. Wake, C. Lima, and P. A. Davies, "Optical generation of millimeterwave signals for fiber-radio systems using a dual mode DFB semiconductor laser," IEEE Trans. Microw. Theory Tech., vol. 43, no. 9, pp. 2270-2276, Sep. 1995.

[9] J. Marti, F. Ramos, V. Polo, J. M. Fuster, and J. L. Corra, "Millimeterwave signal generation and harmonic upconversion through PM-IM conversion in chirped fiber gratings," in Proc. Int. Top. Microw. Photon. Meeting, Nov. 1999, vol. 11, pp. 181-184.

[10] T. Kawanishi, T. Sakamoto, S. Shinada, M. Izutsu, S. Oikawa, and K. Yoshiara, "Low phase noise photonic millimeter-wave generation by using a reciprocating optical modulator," in Proc. Opt. Fiber Commun. Conf., Feb. 2004, vol. 1, Paper WL8.

[11] D. Y. Kim, M. Pelusi, Z. Ahmed, D. Novak, H.-F. Liu, and Y. Ogawa, "Ultrastable millimeter-wave signal generation using hybrid mode locking of a monolithic DBR laser," Electron. Lett., vol. 31, no. 9, pp. 733-734, Apr. 1995.

[12] T. Schneider, M. Junker, and D. Hannover, "Generation of millimeter wave signals by stimulated Brillouin scattering for radio over fiber systems," Electron. Lett., vol. 40, no. 23, pp. 1500-1501, 2004.

[13] J. J. O'Reilly, P. M. Lane, R. Heidemann, and R. Hofstetter, "Optical generation of narrow linewidth millimeter wave signals," Electron. Lett., vol. 28, pp. 2024-2025, 1992.

[14] R. W. Tkach and A. R. Chraplyvy, "Fiber Brillouin amplifiers," Opt. Quantum Electron., vol. 21, pp. S105-S112, 1989.

[15] M. F. Ferreira, J. F. Rocha, and J. L. Pinto, "Analysis of the gain and noise characteristics of fiber Brillouin amplifiers," Opt. Quantum Electron., vol. 26, pp. 35-44, 1994.

[16] T. Schneider, M. Junker, and K. U. Lauterbach, "Theoretical and experimental investigation of Brillouin scattering for the generation of millimeter waves," J. Opt. Soc. Amer. B, Opt. Phys., submitted for publication.

[17] J. W. Shi and C. K. Sun, "Theory and design of a tapered line distributed photodetector," J. Lightw. Technol., vol. 20, no. 11, pp. 1942-1950, Nov. 2002.

[18] M. Nikles, L. Thevenaz, and P. A. Robert, "Brillouin gain spectrum characterization in single-mode optical fibers," J. Lightw. Technol, vol. 15, no. 10, pp. 1842-1851, Oct. 1997.

[19] T. Schneider and M. Junker, "Tunable bandwidth enhancement of Brillouin scattering," Opt. Lett., submitted for publication.

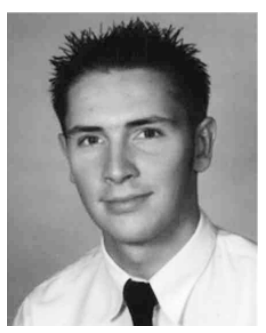

Markus Junker was born in Thuringia, Germany, in 1979. He received the Diploma degree in electrical engineering from the Fachhochschule Leipzig, Leipzig, Germany, in 2004, and is currently working toward the Ph.D. degree at the Dublin Institute of Technology, Dublin, Ireland.

His research interests include the generation of millimeter waves and unconventional amplification systems.

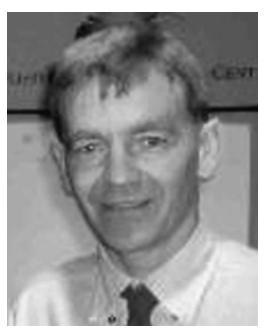

Max James Ammann (M'96) received the Ph.D. degree in microwave antenna design from Trinity College, Dublin, Ireland, in 1997.

He is currently a Senior Lecturer with the School of Electronic and Communications Engineering, Dublin Institute of Technology, Dublin, Ireland. He possesses eight years of industrial experience in radio systems engineering and antenna design. His research interests include electromagnetic theory, wide-band antennas, and specific absorption rate (SAR).

Dr. Ammann is a Chartered Engineer in Ireland. He serves on the International Committee for Electromagnetic Safety (formerly the IEEE Standards Coordinating Committee SCC-28). He is a member of the Royal Irish Academy Committee for Communications and Radio Science. 


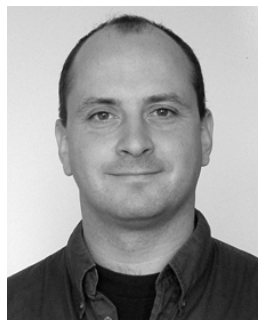

Andreas Thomas Schwarzbacher received the Honors degree in communications engineering from the University of Dieburg, Dieburg, Germany, in 1995, the M.Sc. degree in computer science from the National University of Ireland, Maynooth, Ireland, in 2005, and the Ph.D. degree in electronics from Trinity College, University of Dublin, Dublin, Ireland, in 2001.

He is currently a Lecturer of microelectronics with the Dublin Institute of Technology, Dublin, Ireland. $\mathrm{He}$ has authored or coauthored over 40 publications. Dr. Schwarzbacher is a reviewer for the IEEE JOURNAL OF SOLID-STATE DESIGN and the IEEE TRANSACTIONS ON VERY LARGE SCALE INTEGRATION (VLSI) SYSTEMS. He is also a reviewer for the Institution of Electrical Engineers (IEE), U.K., and for various conferences.

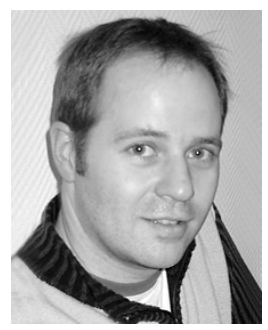

Jens Klinger was born in Saxony, Germany, in 1972. He received the Diploma degree in electrical engineering from the Deutsche Telekom Fachhochschule Leipzig, Leipzig, Germany, in 1998.

Since 2000 , he has been with the Deutsche Telekom Fachhochschule Leipzig, Leipzig, Germany. His research interests are measurement in high-frequency and millimeter-wave systems.

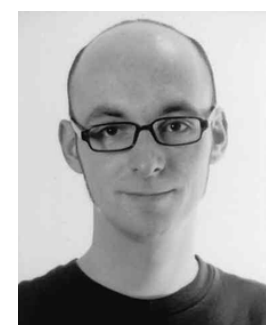

Kai-Uwe Lauterbach was born in Dohna, Germany, in 1978. He received the Diploma degree in electrical engineering from the Deutsche Telekom Fachhochschule Leipzig, Leipzig, Germany, in 2005.

From 1996 to 1998, he trained as a Communication Engineer of telecommunications with Deutsche Telekom AG with distinction from Saxony, Germany. His main research interests are millimeter-wave generation and phase-noise characteristics by SBS and linear and nonlinear high-frequency amplifiers.

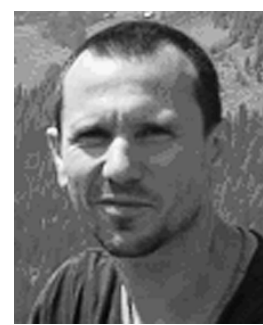

Thomas Schneider was born in Potsdam, Germany, in 1965. He received the Diploma degree in electrical engineering from the Humboldt Universität zu Berlin, Berlin, Germany, in 1995, and the Ph.D. degree in physics from the Brandenburgische Technische Universität Cottbus, Cottbus, Germany, in 2000.

During his doctoral studies, he was engaged in the investigation of nonlinear phenomena induced by an ultrafast refractive index grating. Since 2000, he has been with the Deutsche Telekom Fachhochschule, Leipzig, Germany. His current research interests include wireless communications and nonlinear optics in telecommunications.

Mr. Schneider is a member of the Deutsche Physikalische Gesellschaft and the Optical Society of America (OSA). 